\title{
In Silico Clinical Studies on the Efficacy of Blue Light for Treating Psoriasis in Virtual Patients
}

\author{
Zandra C. Félix Garza, ${ }^{1, *}$ Joerg Liebmann, ${ }^{2}$ Matthias Born, ${ }^{2}$ Peter A.J. Hilbers, ${ }^{1}$ and Natal A.W. van Riel ${ }^{1}$
}

\begin{abstract}
Background: Treatment of psoriasis with blue light reduces the symptoms of this skin condition. Despite the therapeutic effects of blue light, no clear guidelines have been established regarding the irradiation settings. We have previously developed a computational model that enables the study of the impact of the irradiation parameters on the efficacy of this therapeutic approach. This model reproduces the clinically observed response of the average psoriasis patient to irradiation with blue light, but it does not capture the interpatient variations. The inclusion of the interpatient dissimilarities in the model would provide insight into the variability in treatment efficacy among patients.

Methods: In this study, a set of $\sim 500,000$ virtual patients (VPs) that captures the variability in the clinical investigations is generated based on data from two clinical studies and the previously developed model for blue light irradiation of psoriatic skin. First, the minimum number of VPs needed to obtain the same change from baseline (cfb) distribution as in the clinical studies is defined and analyzed. Then, these VPs are used in a series of in silico clinical investigations that explore whether the treatment response of psoriasis patients can be improved by modifying the settings of the therapeutic protocol.

Results: The results suggest that a minimum of $2500 \mathrm{VPs}$ are required to reproduce the cfb distribution observed in vivo. The results from the simulations performed with the VPs predict that the treatment efficacy can be increased for all patients by implementing a therapeutic protocol with daily treatment, and a higher fluence and length of treatment than the currently used settings. These results also suggest that there is no additional benefit to increasing fluence $>200 \mathrm{~J} / \mathrm{cm}^{2}$.
\end{abstract}

Conclusion: This study demonstrates the potential role of in silico clinical studies in the field of dermatology.

Keywords: computational model; epidermis; inflammatory skin conditions; keratinocytes; phototherapy; virtual patients

\section{Introduction}

Studies have shown that irradiating the skin of psoriasis vulgaris patients with blue light is effective in managing this chronic inflammatory skin condition. ${ }^{1,2}$ It decreases the characteristic symptoms of psoriasis, that is, hyperproliferation and reduced differentiation of the structural skin cells known as keratinocytes, ${ }^{3}$ and sustained inflammation, ${ }^{4}$ without the detrimental side effects of the current phototherapy approaches. ${ }^{5,6}$
Despite the beneficial effects of blue light in psoriasis, no clear guidelines have been established regarding the irradiation settings that yield the highest treatment efficacy. The irradiation settings that define the blue light treatment protocols for psoriasis are fluence, expressed in $\mathrm{J} / \mathrm{cm}^{2}$, intensity, defined in $\mathrm{mW} / \mathrm{cm}^{2}$, treatment length, specified in weeks, and frequency of treatment sessions. Clinical investigations ${ }^{1,2,7,8}$ have only studied the impact of specific combinations of

\footnotetext{
${ }^{1}$ Department of Biomedical Engineering, Eindhoven University of Technology, Eindhoven, The Netherlands.

${ }^{2}$ Philips Electronics Netherlands B.V., Research, Eindhoven, The Netherlands.

*Address correspondence to: Zandra C. Félix Garza, PhD, Department of Biomedical Engineering, Eindhoven University of Technology, PO Box 513, Eindhoven, 5600 MB, The Netherlands, E-mail: z.c.felix.garza@tue.nl
}

() Zandra C. Félix Garza et al. 2019 Published by Mary Ann Liebert, Inc. This Open Access article is distributed under the terms of the Creative Commons License (http://creativecommons.org/licenses/by/4.0), which permits unrestricted use, distribution, and reproduction in any medium, provided the original work is properly cited. 
these settings on the treatment efficacy, measured by the change from baseline ( $\mathrm{cfb}$ ) of the local psoriasis severity index (LPSI). However, it is unclear which combination of these irradiation parameters determines the treatment efficacy.

Numerical models enable the analysis of several treatment protocols without the constraints of in vitro and in vivo setups. ${ }^{9,10}$ We have previously developed a computational model for blue light irradiation of psoriatic skin (BLISS) to investigate the impact of the irradiation settings on the efficacy of this therapeutic approach. ${ }^{11}$ The model results suggested that high efficacy is achieved by regimes with long duration and high fluence levels, regardless of the chosen intensity. BLISS describes the clinically observed response of the average mild psoriasis patient to irradiation with blue light but provides no insight on the interpatient variations in treatment response.

Clinical studies ${ }^{1}$ show a large interpatient variability in the treatment response. The variability in treatment response is not exclusive of blue light treatment. It is well known that the available treatments for psoriasis are not entirely effective for all patients. ${ }^{12,13}$ For example, the systemic drug methotrexate, widely used in moderate-to-severe psoriasis, ${ }^{14}$ yields a response lower than the targeted $75 \%$ improvement in the psoriasis area and severity index (PASI), a common measurement used in the clinic, ${ }^{1,2,78}$ for $40-65 \%$ of the treated patients. ${ }^{15}$ The interpatient variability in response to psoriasis treatment has been attributed to the heterogeneity of inflammatory networks driving the disease, ${ }^{16}$ the bimodal activation of the immune system, ${ }^{17}$ and the genetic heterogeneity in the disease. ${ }^{18,19}$

Regardless of the source of this heterogeneity in the treatment response, it is essential that the model accounts for the average patient response as well as the interpatient variability to increase its applicability. One approach to achieve this is to develop alternative parameter sets that reflect the distribution of population-level data and sample the uncertainty in the model parameters. ${ }^{20,21}$ These sets of parameterizations are known as virtual patients (VPs). ${ }^{21-23}$ These VPs are generated from a given model, using prior information on the physiologically plausible range of the parameters and outputs in the model. A parameter set is randomly selected from the predefined parameter space and fed to the model. This parameter set is optimized until the model outputs are physiologically plausible. The process is repeated several times to generate a plausible population. From this plausible population, a final pool of VPs is derived by selecting only those more likely to be included in the real patient population. This selection is optimized such that the final population of VPs is the best possible given the patients in the pool of plausible patients (PPs). ${ }^{21}$

A virtual population (VPop) that reflects individual subject and population-level characteristics of a typical clinical cohort increases the confidence that the prospective simulations of the response to a novel therapy reflect the intersubject variability observed in the clinic. ${ }^{24,25}$

In this study, a set of VPs is generated by fitting the distribution of decrease in disease severity, referred to as $\mathrm{cfb}$, after treatment with blue light from two clinical investigations to the model BLISS. This VPop is then used in a series of in silico clinical studies to explore whether the treatment response of all patients can be improved by modifying the settings used in the therapeutic protocol.

\section{Materials and Methods}

The development of the virtual psoriasis patients and their use in the implementation of in silico clinical studies of blue light treatment was executed in Matlab R2017b (The Mathworks, Inc.). The model BLISS used for this study has been previously described ${ }^{11}$ and can be found in GitHub and the BioModels Database ${ }^{26}$ with identifier BIOMD0000000695. BLISS ${ }^{11}$ is a deterministic model defined by a set of 12 ordinary differential equations that describe the time evolution of the keratinocytes in mild psoriasis as they move vertically through the sublayers of the epidermis while blue light is irradiated on them. The model computes the cell density of the keratinocytes during and after blue light therapy, which allows a direct comparison of the model's results with experimental data.

Clinical data are often shown in terms of the PASI, or its local form, that is, LPSI. ${ }^{1,2,8}$ These indices account for the levels of inflammation, scaling, and induration, and yield a maximum value of 72 . Mild psoriasis, which is the target of blue light therapy, corresponds to a severity index value $\leq 10$. The model results are compared with the clinical data by deriving the LPSI from the relative change in the total cell density of keratinocytes, considering an initial LPSI $\leq 10 .{ }^{11}$

\section{Generation of VPs}

The algorithm used to generate the pool of VPs is shown in Figure 1. The first step to generate the VPs from BLISS is to define the input parameters $p$ that describe a VP and set the boundaries for these parameters. The parameters selected to describe a VP with psoriasis are presented in Table 1 . The values of these parameters were set to an arbitrary range of $\pm 10 \%$ of 


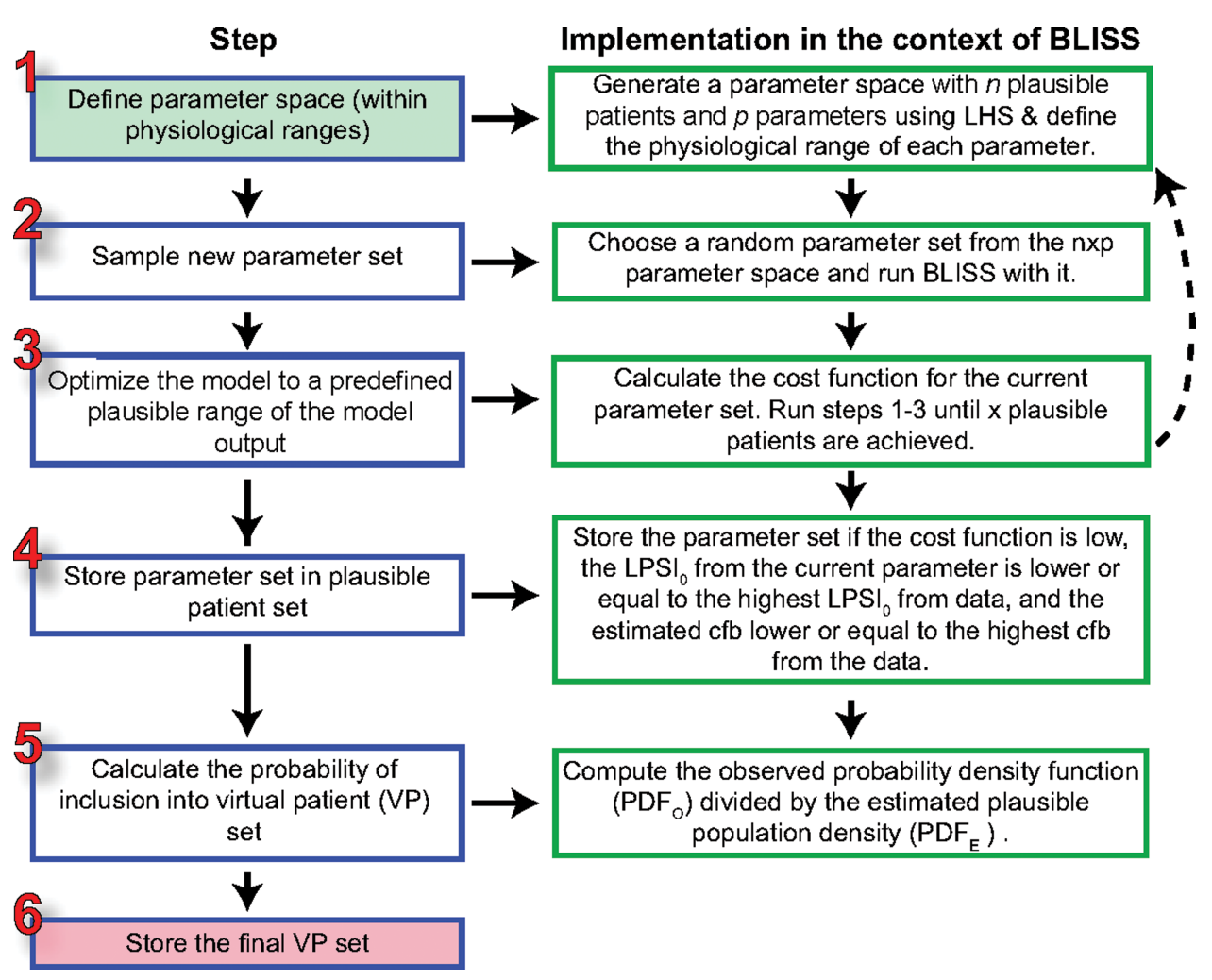

FIG. 1. Overview of the algorithm for generation and selection of VPs. To generate the VPs from BLISS, the physiologically reasonable ranges are defined for the parameter values and model outputs, and the simulation is performed for a given number of PPs. The PPs are stored in the VPop if they comply with the defined requirements. The VPop is built by selecting the population with probability proportional to the prevalence in the real population relative to the incidence in the plausible population. This selection is then stored as the final set of VPs. PPs, plausible patients; VPop, virtual population; VPs, virtual patients.

Table 1. Parameters That Define the Virtual Patients and Their Expected Physiological Ranges

\begin{tabular}{|c|c|c|c|c|}
\hline Parameter & Description & Lower bound & Upper bound & Units \\
\hline $\mathrm{LPSI}_{0}$ & Baseline local psoriasis severity index & 0 & 10 & a.u. \\
\hline$\theta_{\mathrm{BL} \beta}$ & Factor of blue light contribution to cell death & 0 & 0.03 & a.u. \\
\hline $\mathrm{SCO}_{h}$ & Initial cell density of healthy stem cells & 325.8 & 398.2 & Cells per $\mathrm{mm}^{2}$ \\
\hline $\mathrm{TAO}_{h}$ & Initial cell density of healthy transit amplifying cells & 69.3 & 84.7 & Cells per $\mathrm{mm}^{2}$ \\
\hline $\mathrm{GAO}_{h}$ & Initial cell density of healthy growth arrested cells & 54.9 & 67.9 & Cells per $\mathrm{mm}^{2}$ \\
\hline $\mathrm{SPO}_{h}$ & Initial cell density of healthy spinous cells & 214.2 & 261.8 & Cells per $\mathrm{mm}^{2}$ \\
\hline $\mathrm{GCO}_{h}$ & Initial cell density of healthy granular cells & 107.1 & 130.9 & Cells per $\mathrm{mm}^{2}$ \\
\hline $\mathrm{CCO}_{h}$ & Initial cell density of healthy corneocytes & 166.5 & 203.5 & Cells per $\mathrm{mm}^{2}$ \\
\hline $\mathrm{SCO}_{d}$ & Initial cell density of psoriatic stem cells & 5813.1 & 7104.9 & Cells per $\mathrm{mm}^{2}$ \\
\hline $\mathrm{TAO}_{d}$ & Initial cell density of psoriatic transit amplifying cells & 29698.2 & 35307.8 & Cells per $\mathrm{mm}^{2}$ \\
\hline $\mathrm{GAO}_{d}$ & Initial cell density of psoriatic growth arrested cells & 18482.4 & 22589.6 & Cells per $\mathrm{mm}^{2}$ \\
\hline $\mathrm{SPO}_{d}$ & Initial cell density of psoriatic spinous cells & 71809.2 & 87766.8 & Cells per $\mathrm{mm}^{2}$ \\
\hline $\mathrm{GCO}_{d}$ & Initial cell density of psoriatic granular cells & 0 & 0 & Cells per $\mathrm{mm}^{2}$ \\
\hline $\mathrm{CCO}_{d}$ & Initial cell density of psoriatic corneocytes & 69869.7 & 85396.3 & Cells per $\mathrm{mm}^{2}$ \\
\hline$\rho s c$ & Fold change of proliferation rate in psoriasis stem cells & 3 & 5 & a.u. \\
\hline pta & Fold change of proliferation rate in psoriasis transit amplifying cells & 3 & 5 & a.u. \\
\hline$\rho t r$ & Fold change of psoriasis cells transit rate & 4 & 6 & a.u. \\
\hline$\rho d e$ & Fold change of desquamation rate in psoriasis corneocytes & 3 & 5 & a.u. \\
\hline Ald & Epidermal apoptosis index for psoriatic skin & 0.03 & 0.05 & $\%$ \\
\hline$\lambda$ & Fold change of stem cells growth capacity in psoriasis & 2.5 & 4.5 & a.u. \\
\hline
\end{tabular}

a.u., arbitrary units. 
their original value ${ }^{11}$ except for the baseline LPSI $\left(\mathrm{LPSI}_{0}\right)$, whose upper limit was based on the maximum LPSI value for mild psoriasis. Once these parameters have been defined, an $n$ number of PPs are made using the Latin hypercube sampling method. From this plausible population, a randomly selected PP is used to run the model of blue light treatment for psoriasis.

For each PP, the cost function $g(p)$ is computed for every parameter defining the patient $\left(M_{n} ;\right.$ Eq. 1$)$, taking into account its lower $\left(l_{n}\right)$ and upper $\left(u_{n}\right)$ bounds. This cost function is based on the sum of squared errors, typically used in optimization problems. The process is repeated for an $n$ number of $P P s$.

$$
g(p)=\sum_{n} \max \left[\left(M_{n}(p)-\frac{l_{n}+u_{n}}{2}\right)^{2}-\left(\frac{u_{n}}{2}-\frac{l_{n}}{2}\right)^{2}\right]
$$

Each PP is then stored if its cost function is lower or equal to the predefined threshold based on the original model, its LPSI $_{0 \mathrm{PP}}$ is lower or equal to the maximum LPSI ${ }_{0}$ observed in the clinical investigations, and its cfb does not exceed the maximum $\mathrm{cfb}$ from the clinical studies. BLISS does not explicitly yield the $\mathrm{cfb}$; however, it can be computed as the difference between the initial $L P S I_{O}$ and the predicted LPSI at the end of the treatment $L P S I_{12}$ (Eq. 2).

$$
c f b=L P S I_{0}-L P S I_{12}
$$

The selected PPs are then used to calculate the probability of inclusion ( $\left.P_{\text {inclusion }}\right)$ of the PPs into the VPop (Eq. 3). This probability is computed from both the empirically observed distribution $\left(\mathrm{PDF}_{0}\right)$ and the density of PPs $\left(\mathrm{PDF}_{\mathrm{E}}\right)$. Both the empirically observed distribution and the density of PPs are calculated based on histograms of the $\mathrm{cfb}$, with a univariate approach, and fitted distribution models. The selected PPs become the VPop if the $P_{\text {inclusion }}$ is $>0.95$. Otherwise, the inclusion rate is optimized until the results for the selected PPs match the data from the real patients.

$$
P_{\text {inclusion }}=\frac{P D F_{O}}{P D F_{E}}
$$

\section{Clinical data}

Data from two clinical investigations of blue light as treatment of psoriasis, referred to as $\mathrm{CT} 02^{1}$ and CT03, ${ }^{7}$ were used to generate the VPs. The number of patients included in each study was 47 for CT02 and 51 for CT03. ${ }^{1,7}$ Both cohorts comprised adult female and male patients with an age ranging from 18 to 75 years. ${ }^{1,7}$
In both studies, blue light was locally applied on the lesions of mild psoriasis patients for 12 weeks with a fluence of either $45 \mathrm{~J} / \mathrm{cm}^{2}$ in CT03, referred to as CT03-45, or $90 \mathrm{~J} / \mathrm{cm}^{2}$ in CT02 ${ }^{1}$ and CT03, ${ }^{7}$ referred to as CT03-90. To assess the efficacy of the therapy, the LPSI was measured at weeks $0,2,4,8$, and 12 . In addition, the $\mathrm{cfb}$ was calculated for each of these time points. The patients from these cohorts had an initial LPSI $<10$. The $\mathrm{cfb}$ of these patients at the end of the 12 weeks had a normal distribution. The maximum $\mathrm{cfb}$ observed at the end of the treatment was 6 for CT02 and CT03-45, and 5 for CT03-90.

\section{Minimum size of the initial pool of PPs}

We investigated the minimum number of initial PPs needed to obtain the same $\mathrm{cfb}$ distribution as in the clinical investigations. This number was defined as the initial pool size whose final population of VPs would yield a probability density function (pdf) ratio with a variation $\leq 0.01 \mathrm{U}$ compared with larger initial pools. Ten pools of PPs were used, ranging in size from 80 to 1 million initial PPs (Fig. 2A-D).

\section{In silico clinical studies on the impact of therapeutic} settings on the treatment efficacy of blue light The pool of VPs was used in combination with BLISS in a series of in silico clinical studies to predict whether the effectiveness of blue light irradiation as a treatment for psoriasis can be increased by using a combination of therapeutic settings different from that currently used in the protocol of the clinical investigations. ${ }^{1,7}$ Sets of simulations were implemented for fluence, length of treatment, and frequency of treatment sessions (Table 2). Depending on the analyzed parameter, the rest of the therapeutic settings were kept at a constant value for fluence, length of treatment, and frequency of treatment sessions. These values were $90 \mathrm{~J} /$ $\mathrm{cm}^{2}, 84$ days, and daily sessions, respectively.

\section{Results}

Generation of the VPop and the minimum size of the initial pools

Three pools of $\sim 500,000$ VPs were generated with population-level characteristics that match those from CT02 and CT03. The data from CT03 were further divided into the two groups of treatment used in the original clinical investigation, that is, one with treatment at a fluence of $45 \mathrm{~J} / \mathrm{cm}^{2}$ (CT03-45) and the other where $90 \mathrm{~J} / \mathrm{cm}^{2}$ of blue light was applied on the skin of the patients (CT03-90). These three pools of VPs were 

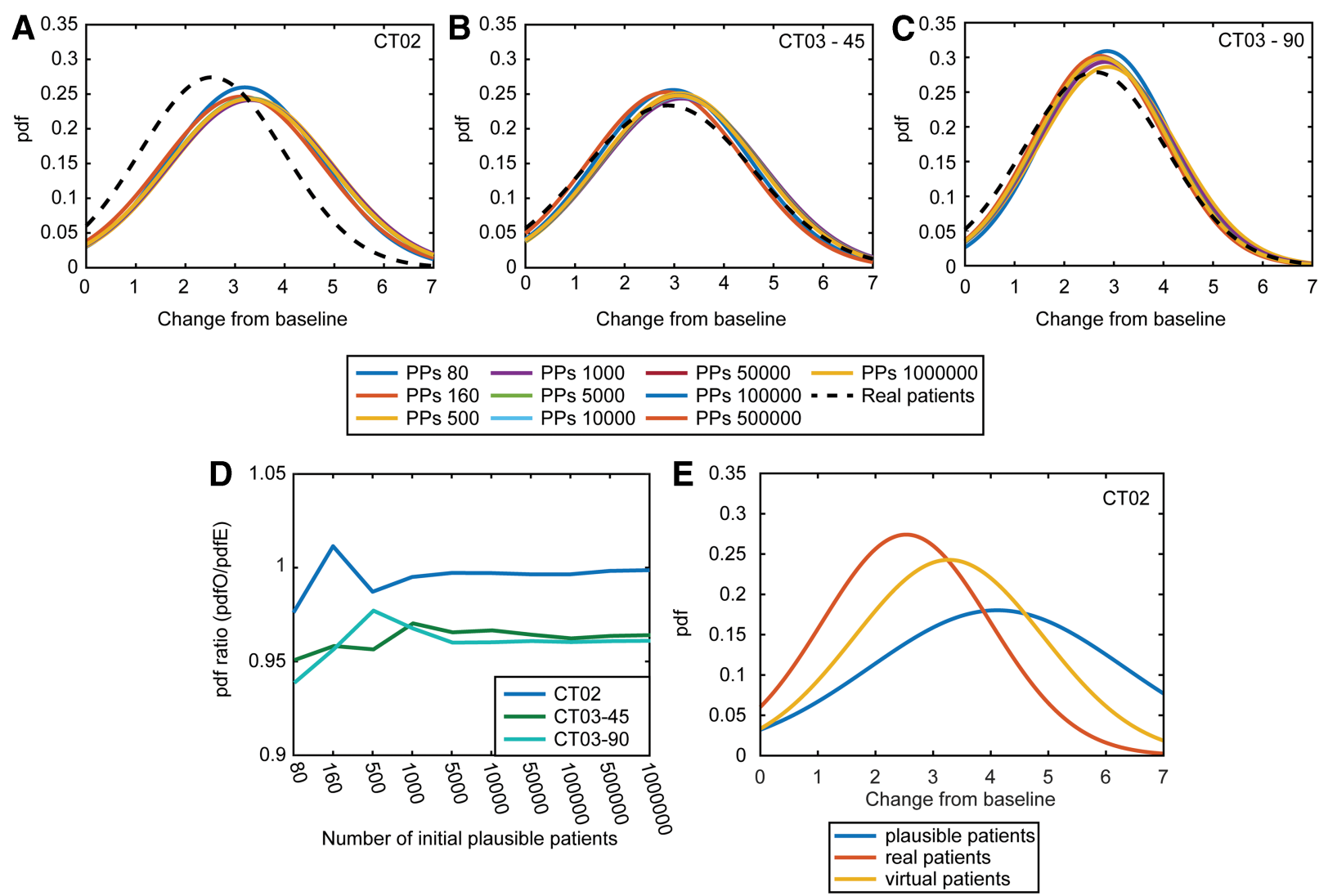

FIG. 2. Analysis of three sets of VP populations with a varying initial number of patients. (A-C) The pdf of the $\mathrm{cfb}$ in the final VPs obtained from an initial pool of PPs with size $n$ is plotted for the three sets of VPs, that is, CT02, CT03-45, and CT03-90. These pdfs are compared with each other and the pdf of the real patients (dashed black line) in the two clinical investigations. (D) The ratio of the $\mathrm{PDF}_{\mathrm{O}}$ to the $\mathrm{PDF}_{\mathrm{E}}$ from the VPs obtained from the initial pools of PPs with size $n$ is derived for CT02 (blue), CT03-45 (green), and CT03-90 (turquoise; see Eq. 3). (E) The pdf of the cfb of the $\sim 500,000$ VPs (yellow) for CT02 is compared with the pdf of the 1,000,000 initial PPs (blue) and the real patients (red). cfb, change from baseline; pdf, probability density function; $\mathrm{PDF}_{\mathrm{E}}$, estimated probability density function; $\mathrm{PDF}_{\mathrm{O}}$, observed probability density function.

Table 2. Virtual Clinical Studies Implemented for Fluence, Length of Treatment, and Frequency of Treatment Sessions

\begin{tabular}{|c|c|c|}
\hline No. & Tested parameter & Value \\
\hline 1 & Fluence & $15-500 \mathrm{~J} / \mathrm{cm}^{2}$ \\
\hline 2 & Length of treatment & 14-140 days \\
\hline 3 & $\begin{array}{l}\text { Frequency of } \\
\text { treatment sessions }\end{array}$ & $\begin{array}{l}\text { 1. One-third of treatment length as daily } \\
\text { treatment, followed by two-third of } \\
\text { every other day treatment sessions. } \\
\text { 2. Two-third of treatment length as daily } \\
\text { treatment, followed by one-third of } \\
\text { every other day treatment sessions. } \\
\text { 3. Daily sessions. } \\
\text { 4. Every other day sessions. }\end{array}$ \\
\hline
\end{tabular}

derived from three initial pools of 1 million PPs, using the algorithm described in Figure 1 (Fig. 2A-C). Furthermore, we determined the minimum size of the initial pools of PPs that would lead to the same $\mathrm{cfb}$ distribution observed in the clinical investigations.

The minimum initial pool size was found to be 5000 for both CT02 and CT03 (Fig. 2D). As expected, neither of the initial plausible populations matched the cfb pdf of CT02, CT03-45, and CT03-90, regardless of the number of PPs in the pool (Fig. 2E). However, after filtering out the initial PPs that had a cost function lower or equal to the predefined threshold and whose $L P S I_{O P P}$ and $c f b$ exceeded those observed in the clinical studies, it was established that approximately half of 
the initial PPs are unlikely to be in the final distribution (Fig. 2E). Hence, when starting with 1 million PPs, the final pool of VPs only includes $\sim 500,000$ patients.

Analyzing the parameters in the final pools of VPs for CT02, CT03-45, and CT03-90, minor differences were observed among them (Supplementary Figs. S1 and S2). This suggested that the same pool of VPs could be used to implement in silico clinical investigations of both clinical studies regardless of the fluence used in the therapeutic protocol. Furthermore, these VPs could also be used to define in silico clinical studies where the therapeutic settings are analyzed.

\section{In silico clinical studies assessing the impact of the} irradiation settings on the response to treatment The pool of VPs was used in combination with BLISS in a series of in silico clinical studies on the therapeutic settings used for the treatment of psoriasis with blue light (Fig. 3). In these simulations, the impact of the fluence (Fig. 3A-D), the frequency of the treatment sessions (Fig. 3E-H), and the length of treatment (Fig. 3I-K) on the therapeutic efficacy, defined as the relative decrease in the LPSI, was analyzed for all VPs.

The results from these simulations suggest that the treatment efficacy can be increased for all patients, including those with low treatment response. This can be achieved by implementing a therapeutic protocol with daily treatment and a higher fluence than the currently used settings. These results are consistent with the model predictions from Félix Garza et al. ${ }^{11}$ and extend them by showing the outcome for the whole patient population instead of just the average.

The results from the digital clinical studies on the fluence (Fig. 3A-D) suggest that increasing fluence would lead to a higher treatment efficacy reaching a plateau $>200 \mathrm{~J} / \mathrm{cm}^{2}$. The predictions on the frequency of treatment sessions (Fig. 3E-H) indicate that although increasing the number of sessions per week results in a higher treatment efficacy than every other day treatment or the combination of the two, the difference is minimal. Finally, the results from the series of simulations on the length of treatment (Fig. 3I-K) suggest that at fluences $\geq 90 \mathrm{~J} / \mathrm{cm}^{2}$, a minimum of 12 weeks or 84 days are needed to reach a high treatment efficacy for all patients. Above this treatment length, small differences in the predicted treatment efficacy are observed.

\section{Discussion}

The main purpose of this mechanistic model of blue light as treatment for psoriasis, that is, BLISS, is to simulate the effect of a certain therapeutic protocol on the treatment efficacy. We have previously shown that this model reproduces the average response of a patient cohort to blue light therapy. ${ }^{11}$ However, predicting only the average response does not provide any insight into the variability of the treatment efficacy among the patients. Furthermore, accounting for the heterogeneity of the patients increases the confidence in the simulation results. ${ }^{21}$

In this study, a methodology is described to generate groups of VPs with outcome parameters similar to those of the cohorts used in clinical investigations of blue light as treatment for psoriasis. ${ }^{1,7}$ The results of simulations performed with these VPs show the potential application of this approach to explore the effect of interpatient variability in the treatment efficacy of blue light therapy.

An attractive aspect of the approach used in this study to develop the VPs is that, due to its probabilistic nature, once the population of VPs is generated, it can be used to define subpopulations that match the characteristics of a given pool of real patients. Furthermore, the similarities in the parameter sets that represent the groups of VPs derived from three sets of real patients (Fig. 2; Supplementary Figs. S1 and S2) suggests that the same pool of VPs can be used in a wide variety of in silico clinical studies of blue light as treatment for psoriasis.

Currently, there is no agreement on how VPs should be created, some use Markov Chain Monte Carlo algorithms, ${ }^{21,27}$ whereas others propose the weighting of model parameters and outputs. ${ }^{28}$ However, the consensus is that the ensemble of VPs should reflect the distribution of the population-level data. As shown in Figure 2, the steps used to create the VPs in this study lead to a good description of the clinical data.

The main advantage of the algorithm described in this study, compared with others, ${ }^{25,28}$ is that it yields a pool of VPs that closely matches the available data while keeping a low bias level and avoiding skewing the simulation results. Unlike the approach proposed by Klinke et al., ${ }^{28}$ it avoids overweighting unrealistic model solutions. Furthermore, our approach does not comprise any assumption on the correlation between parameters in the population of interest beyond those supported by the available clinical data.

The definition of the parameters in the BLISS model, ${ }^{11}$ used in this study to generate the VPs and implement the clinical studies, involved thorough local and multiple parameter sensitivity analyses. This resulted in a model that accurately matched the reported average therapeutic outcome of blue light therapy. However, those sensitivity 


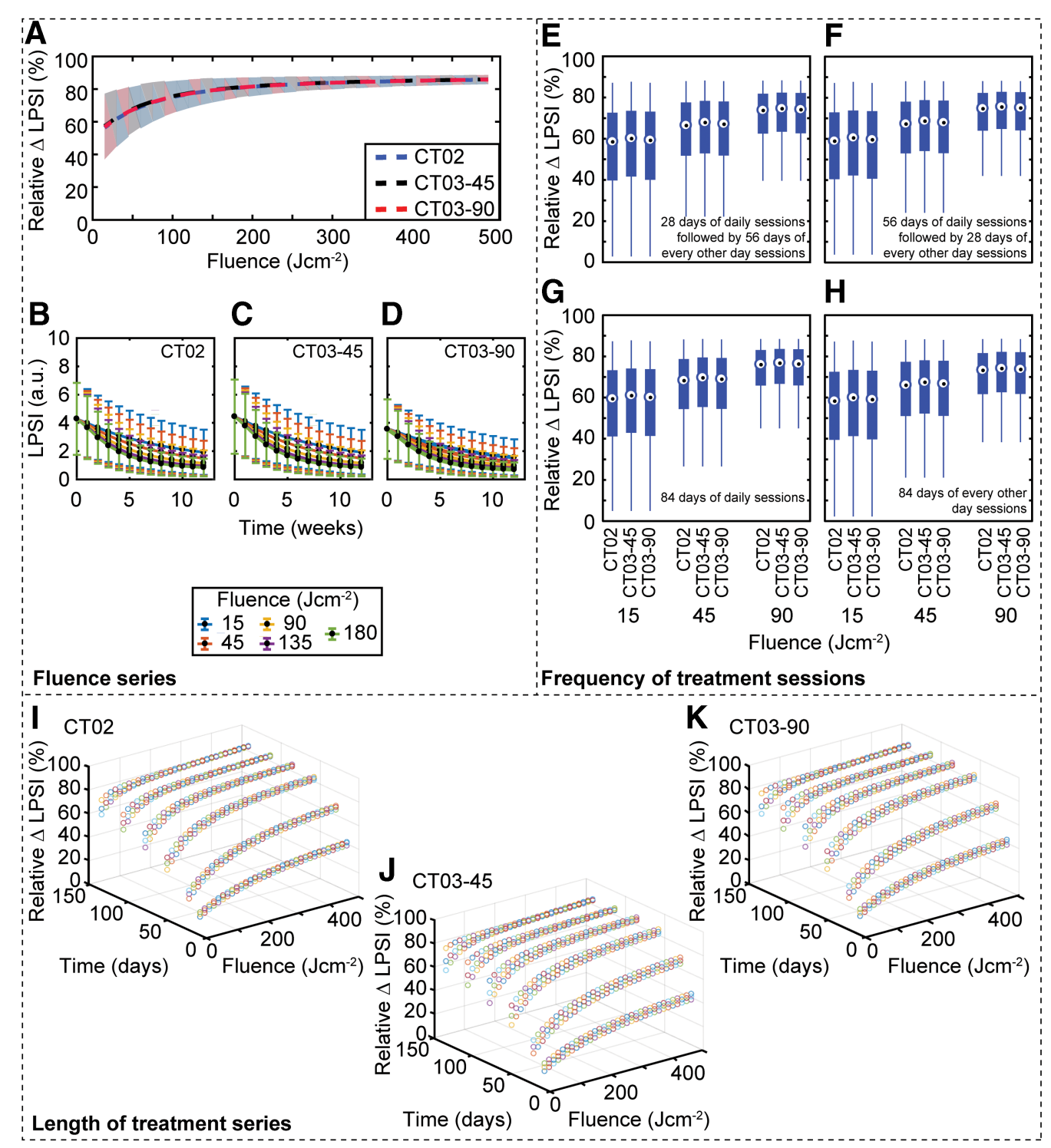

FIG. 3. In silico clinical studies on the fluence, the frequency of irradiation sessions, and the length of treatment. A series of computer-based clinical investigations are implemented using BLISS and the three pools of VPs to assess the effect of fluence $(\mathbf{A})$, the number of sessions $(\mathbf{E}-\mathbf{H})$, and the length of treatment (I-K) on the treatment efficacy of blue light irradiation, which is defined by the relative decrease in the LPSI. (A) Simulations are performed for 12 weeks blue light treatment with daily treatment sessions and a dose ranging from 15 to $500 \mathrm{~J} /$ $\mathrm{cm}^{2}$ using each of the ensembles of VPs, that is, CT02 (dashed blue line), CT03-45 (dashed black line), and CT03-90 (dashed red line). The relative decrease in the LPSI is derived for all VPs in each in silico clinical study for the fluence setting. The colored region in the plot represents the standard deviation for all cases. (B-D) In addition to the relative decrease in the LPSI, the week-wise decrease in the LPSI is predicted for CT02 (B), CT03-45 (C), and CT03-90 (D), using a fluence of $15 \mathrm{~J} / \mathrm{cm}^{2}$ (blue line), $45 \mathrm{~J} / \mathrm{cm}^{2}$ (red line), $90 \mathrm{~J} / \mathrm{cm}^{2}$ (yellow line), $135 \mathrm{~J} / \mathrm{cm}^{2}$ (purple line), and $180 \mathrm{~J} / \mathrm{cm}^{2}$ (green line). (E-H) The boxplots in these panels depict the digital clinical studies implemented using the three available pools of VPs, for four possible cases on the frequency of treatment sessions considering a treatment of 12 weeks with a dose of 15,45 , and $90 \mathrm{~J} / \mathrm{cm}^{2}$. On each box, the central round mark represents the median. The lines on the top and bottom of each box encompass the most extreme data points not considered outliers. The four tested cases are: (E) The first third of the treatment with daily treatment sessions, followed by two-thirds of every other day treatment; (F) Two-thirds of the treatment with daily sessions, followed by one-third of every other day treatment sessions; (G) Twelve weeks of daily treatment; $(\mathbf{H})$ Twelve weeks of every other day treatment. (I-K) Simulations performed for a treatment protocol with a varying treatment length of 14-140 days and a fluence ranging from 15 to $500 \mathrm{~J} / \mathrm{cm}^{2}$ are described in three-dimensional plots for the VPs of CT02 (I), CT03-45 (J), and CT03-90 (K). a.u., arbitrary units; LPSI, local psoriasis severity index. 
analyses alone could not enable the model to yield predictions for the whole population of patients included in the clinical studies. The pools of VPs generated with our algorithm along with a more thorough analysis of the parameters used in the BLISS model ${ }^{11}$ increase the confidence in the model predictions beyond those made for the average patient.

The series of in silico clinical investigations (Fig. 3) implemented based on BLISS and the VPs suggest the same trends described by Félix Garza et al. ${ }^{11}$ However, in contrast with their results, our predictions are made based on a large population of VPs. In both this study and the study of Félix Garza et al., ${ }^{11}$ increasing the length of the treatment and the fluence of blue light applied on the skin of a given patient would yield a higher response to the therapy reflected in a lower severity index.

The results presented in Figure 3 show a quick saturation on the fluence, reaching a plateau $\sim 200 \mathrm{~J} / \mathrm{cm}^{2}$, well below the fluence at which detrimental effects are induced on the keratinocytes, that is, $500 \mathrm{~J} / \mathrm{cm}^{2}{ }^{29}$ This observation suggests that there is no additional benefit to increasing this therapeutic setting $>200 \mathrm{~J} /$ $\mathrm{cm}^{2}$. Future clinical studies should explore whether the upper bound of this therapeutic setting is in the range of the predicted value. The duration of the treatment seems to cover a wider range before it saturates, which indicates that blue light therapy can be used for a long period of time. Currently, no long-term treatment data are available on this therapeutic approach. Additional clinical investigations are needed to determine the upper limit of this therapeutic setting.

Compared with the predictions of Félix Garza et al., ${ }^{11}$ the results described in this study predict a more optimistic response to the treatment for all therapeutic settings, including those from the clinical investigation of Pfaff et al. ${ }^{1}$ Nevertheless, the predictions for the total population are still within the confidence interval reported in the original clinical investigation. A possible explanation for this discrepancy is the inclusion of the apoptosis factor (Table 1) $\theta_{B L \beta}$ in the set of parameters that define each VP, which was not considered by Félix Garza et al. ${ }^{11}$ To verify this, future work should include the repetition of the simulations described in this study with a fixed fluence-dependent $\theta_{B L \beta}$.

Regardless of the over-optimistic predictions, these series of virtual clinical investigations demonstrate the use of BLISS in the systematic analysis of the impact of the therapeutic settings in the treatment efficacy of blue light therapy for psoriasis. The VP population here described resembles the pool of patients commonly used in clinical studies of blue light therapy for mild psoriasis. Furthermore, the predictions made by implementing simulations with them are insightful for the therapeutic settings that may be most adequate for a given patient.

However, our approach has two main limitations. First, the filters used to discern between acceptable and unacceptable parameter sets are not fully based on biological observations due to the limited available data on these parameters. Second, the upper and lower bounds of each parameter were defined as $\pm 10 \%$ of the original parameter. This may result in a large range for some parameters and a narrow range for others. Owing to the nature of the model parameters, limited information is available as to what the most adequate bounds are for each parameter used to define the VPs.

Future research should study the long-term, mild psoriasis-specific, and blue-light changes induced in proliferation, differentiation, and cell death of keratinocytes in skin samples obtained from a large population. These data would provide additional insight into the characteristics of the population and the lower and upper bounds of the parameters used to define our VPs. The pools of VPs here described can only be used for simulations of treatments targeting mild psoriasis, particularly blue light therapy. Their application to other therapeutic approaches would require changes to the BLISS model, ${ }^{11}$ the values of the model parameters, and the bounds of the physiologically plausible model output.

\section{Conclusions}

This study contributes to the systematic selection of the adequate blue light treatment protocol for a specific population of psoriasis patients. It enables the current model of blue light irradiation for psoriasis to reflect the intersubject variability typically observed in clinical investigations and proposes the use of in silico clinical studies in the field of dermatology, particularly for chronic inflammatory skin diseases.

\section{Authors' Contributions}

Z.C.F.G. wrote the article. Z.C.F.G., J.L., M.B., P.A.J.H., and N.A.W.v.R. conceived and designed the computational framework. Z.C.F.G. implemented the framework and performed the simulations. Z.C.F.G. performed the computational analysis. J.L., M.B., P.A.J.H., and N.A.W.v.R. supervised the study. All authors read and approved the final article. This article has been submitted solely to the Systems Medicine journal and is not published, in press, or submitted elsewhere. 


\section{Authors Disclosure Statement}

M.B. and J.L. are paid employees of Philips Electronics Netherlands B.V., Research.

\section{Supplementary Material \\ Supplementary Figure S1 \\ Supplementary Figure S2}

\section{References}

1. Pfaff S, Liebmann J, Born $M$, et al. Prospective randomized long-term study on the efficacy and safety of UV-free blue light for treating mild psoriasis vulgaris. Dermatology. 2015;231:24-34.

2. Weinstabl A, Hoff-Lesch S, Merk HF, et al. Prospective randomized study on the efficacy of blue light in the treatment of psoriasis vulgaris. Dermatology. 2011;223:251-259.

3. Liebmann J, Born M, Kolb-Bachofen V. Blue-light irradiation regulates proliferation and differentiation in human skin cells. J Invest Dermatol. 2010;130:259-269.

4. Shnitkind E, Yaping E, Geen S, et al. Anti-inflammatory properties of narrow-band blue light. J Drugs Dermatol. 2006;5:605-610.

5. de Gruijl FR. For better or for worse, UV in psoriasis. Exp Dermatol. 2016; 25:945-946.

6. Kunisada $M$, Kumimoto $H$, Ishizaki $K$, et al. Narrow-band UVB induces more carcinogenic skin tumors than broad-band UVB through the formation of cyclobutane pyrimidine dimer. J Invest Dermatol. 2007;127: 2865-2871.

7. ClinicalTrials.gov. Efficacy and safety of blue light $(453 \mathrm{~nm})$ treatment for mild psoriasis vulgaris over three months compared to Vitamin D. https:// clinicaltrials.gov/ct2/show/NCT02735187. Accessed May 2, 2019.

8. Maari C, Viau G, Bissonnette R. Repeated exposure to blue light does not improve psoriasis. J Am Acad Dermatol. 2003;49:55-58.

9. Diffey B. Towards optimal regimens for the UVB phototherapy of psoriasis: a mathematical model. Acta Derm Venereol. 2004;84:259-264.

10. Weatherhead SC, Farr PM, Reynolds NJ. Spectral effects of UV on psoriasis. Photochem Photobiol Sci. 2013;12:47-53.

11. Félix Garza ZC, Liebmann J, Born M, et al. A dynamic model for prediction of psoriasis management by blue light irradiation. Front Physiol. 2017;8:28.

12. Woolf RT, Smith $\mathrm{CH}$. How genetic variation affects patient response and outcome to therapy for psoriasis. Expert Rev Clin Immunol. 2010;6:957-966.

13. Ryan $C$. Identification of pharmacogenetic markers of treatment response to biologic therapies in psoriasis—is there a benefit? Br J Dermatol. 2017; 176:1127-1128.

14. Menter A, Korman NJ, Elmets CA, et al. Guidelines of care for the management of psoriasis and psoriatic arthritis: section 3. Guidelines of care for the management and treatment of psoriasis with topical therapies. J Am Acad Dermatol. 2009;60:643-659.

15. Saurat J-H, Stingl G, Dubertret L, et al. Efficacy and safety results from the randomized controlled comparative study of adalimumab vs. methotrexate vs. placebo in patients with psoriasis (CHAMPION). Br J Dermatol. 2008;158:558-566.

16. Swindell WR, Xing $X$, Stuart PE, et al. Heterogeneity of inflammatory and cytokine networks in chronic plaque psoriasis. PLoS One. 2012;7:e34594

17. Albanesi $C$. Heterogeneity of psoriasis and bimodal activation of local immune responses. Br J Dermatol. 2014;170:7-8.
18. Yin $X$, Low HQ, Wang $L$, et al. Genome-wide meta-analysis identifies multiple novel associations and ethnic heterogeneity of psoriasis susceptibility. Nat Commun. 2015;6:6916.

19. Reek JMPA, Coenen MJH, Arias ML, et al. Polymorphisms in CD84, IL12B and TNFAIP3 are associated with response to biologics in patients with psoriasis. Br J Dermatol. 2017;176:1288-1296.

20. Hallow KM, Lo A, Beh J, et al. A model-based approach to investigating the pathophysiological mechanisms of hypertension and response to antihypertensive therapies: extending the Guyton model. Am J Physiol Regul Integr Comp Physiol. 2014;306:R647-R662.

21. Allen $R$, Rieger $T$, Musante $C$. Efficient generation and selection of virtual populations in quantitative systems pharmacology models. CPT Pharmacometrics Syst Pharmacol. 2016;5:140-146.

22. Viceconti M, Henney A, Morley-Fletcher E. In silico clinical trials: how computer simulation will transform the biomedical industry. Int J Clin Trials. 2016;3:37-46.

23. Rieger TR, Allen RJ, Bystricky L, et al. Improving the generation and selection of virtual populations in quantitative systems pharmacology models. Prog Biophys Mol Biol. 2018;139:15-22.

24. Lawson BAJ, Drovandi CC, Cusimano N, et al. Unlocking data sets by calibrating populations of models to data density: a study in atrial electrophysiology. Sci Adv. 2018;4:e1701676.

25. Schmidt BJ, Casey FP, Paterson T, et al. Alternate virtual populations elucidate the type I interferon signature predictive of the response to rituximab in rheumatoid arthritis. BMC Bioinformatics. 2013;14:221.

26. Chelliah V, Laibe $C$, Le Novère $N$. BioModels database: a repository of mathematical models of biological processes. In: In Silico Systems Biology. (Schneider MV; ed.). Humana Press, Totowa, NJ, 2013. pp. 189-199.

27. Andrieu C, Thoms J. A tutorial on adaptive MCMC. Stat Comput. 2008;18: 343-373.

28. Klinke DJ. Integrating epidemiological data into a mechanistic model of type 2 diabetes: validating the prevalence of virtual patients. Ann Biomed Eng. 2008;36:321-334.

29. Awakowicz P, Bibinov N, Born M, et al. Biological stimulation of the human skin applying health promoting light and plasma sources. Contrib Plasm Phys. 2009;49:641-647.

Cite this article as: Félix Garza ZC, Liebmann J, Born M, Hilbers PAJ, van Riel NAW (2019) In silico clinical studies on the efficacy of blue light for treating psoriasis in virtual patients, Systems Medicine 2:1, 10-18, DOI: 10.1089/sysm.2018.0007.

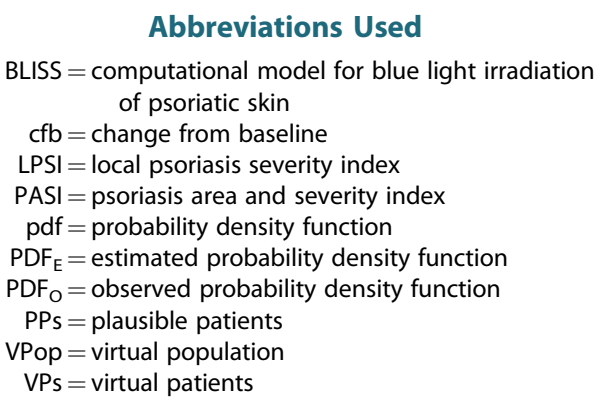

